\title{
Première recherche du modèle de « mohe » en milieu interculturel - cas des entreprises sino- françaises installées en Chine
}

\section{Zhijie Wang}

\section{(2) OpenEdition}

\section{Journals}

Édition électronique

URL : http://journals.openedition.org/communicationorganisation/2600

DOI : 10.4000/communicationorganisation. 2600

ISSN : 1775-3546

\section{Éditeur}

Presses universitaires de Bordeaux

\section{Édition imprimée}

Date de publication : 1 novembre 2001

ISSN : 1168-5549

\section{Référence électronique}

Zhijie Wang, «Première recherche du modèle de « mohe » en milieu interculturel - cas des entreprises sino-françaises installées en Chine », Communication et organisation [En ligne], 20 | 2001, mis en ligne le 27 mars 2012, consulté le 04 mai 2019. URL : http://journals.openedition.org/ communicationorganisation/2600 ; DOI : 10.4000/communicationorganisation.2600

Ce document a été généré automatiquement le 4 mai 2019.

(c) Presses universitaires de Bordeaux 


\title{
Première recherche du modèle de « mohe » en milieu interculturel - cas des entreprises sino-françaises installées en Chine
}

\author{
Zhijie Wang
}

1 Avec la mondialisation de l'économie, de plus en plus d'entreprises interculturelles se développent au niveau transcontinental. Cette évolution commerciale et industrielle donne naissance à un creuset social où se confrontent, voire s'affrontent, des personnes, des groupes et des cultures qui pensent, sentent et agissent différemment. Dans ce cas-là, une coopération harmonieuse serait primordiale pour toute autre réussite. Est-ce possible alors de réaliser une telle coopération ? Des esprits différents pourront-ils coexister pour régler en commun des problèmes confrontés ? Comment doit-on envisager de tels sujets et agir au mieux en vue d'une telle coexistence? Au-delà des expériences réussies et en cours, il est intéressant d'observer les phénomènes et les problèmes de différents points de vue épistémologiques et philosophiques.

2 Notre réflexion, focalisée notamment sur les entreprises interculturelles sino-françaises, est initialement inspirée par un mot chinois « mohe». Le «mohe » est un terme courant en Chine. Quand on parle d'un nouveau mariage, ce terme est souvent évoqué : les nouveaux mariés passeront sûrement une étape courte ou longue, réussie ou ratée de « mohe ». En chinois, mo, c'est dans le sens de moudre, de limer, de frotter tandis que he, dans le sens de s'adapter, de s'harmoniser, de s'unir. Un seul mot «mohe » combine ces deux sens comprenant ainsi tout le processus de véritable union d'un ménage. Evidemment, un ménage est bien différent d'une entreprise mixte. Pour cette dernière, les problèmes se posent en principe plus sous deux autres dimensions : organisationnelle et culturelle. Donc beaucoup plus compliqué. En concrétisant notre problématique cidessus, une série de questions se posent au fur et à mesure : est-ce qu'il existe un modèle de «mohe » dans la coopération interculturelle? Si oui, quels sont les problèmes exacts existant dans le «mohe»? Peut-on trouver une approche appropriée? Quels sont les 
enjeux importants et les étapes éventuelles de ce «mohe »? Nouvelle époque, nouvelle problématique, tout cela nous invite à une recherche assidue. Le présent travail essaie d'y apporter quelques réflexions, analyses et remarques à partir de la réalité de la coopération interculturelle sino-française en Chine et des approches épistémologiques et philosophiques principalement chinoises.

\section{Problèmes : « joindre les deux bouts » ${ }^{1}$}

3 Si l'on parle des nouvelles entreprises transnationales au lieu d'un couple, le "mohe » réside plutôt dans les chocs de cultures. On entend souvent des plaintes dans des entreprises interculturelles sino-françaises: la partie française ne comprend pas pourquoi les collègues chinois dépensent autant d'argent pour des relations externes; et les directeurs chinois ne sont pas contents non plus que les ingénieurs français soient tellement autoritaires... D'après une statistique introduite dont l'ouvrage de PeillSchoeller sur la gestion interculturelle fait état, $70 \%$ des directeurs généraux des entreprises interculturelles ont vu, en premier lieu, des effets négatifs dans la coopération interculturelle. À part des problèmes financiers, politiques et personnels, ceux relatifs aux chocs de cultures, il faut le reconnaître, sont certainement majeurs. Récemment, un PDG français, ayant travaillé à plusieurs reprises dans des entreprises sino-françaises en Chine, a donné une interprétation intéressante à propos de « Qing-LiFa » et "Li-FaQing »'. Il a dit: "Essentiel pour nous, le Li (logique) ne vient qu'en deuxième chez nos partenaires chinois ". Il a compris après sa phase d'observation que, dans un pays où le Qing joue un si grand rôle, la force d'un produit s'appuie sur celle des hommes qui l'ont réalisé. Il a fait en plus une comparaison de mentalités occidentale et chinoise suivant l'ordre différent de ces trois mots : l'ordre « Li (logique ou raisonnement) - Fa (loi ou règlements) - Qing (affectif ou Relations) exprime la mentalité occidentale tandis que l'ordre » Qing (affectif ou relations) - Li (logique ou raisonnement) - Fa (loi ou règlements), la mentalité chinoise.

On peut dire que ses remarques sont en quelque sorte véritable et intéressante. Il est vrai que pour les Chinois "Qing" est très important dans la plupart des cas. Dans le commerce on garde toujours ces devises: "La bonne entente fait la bonne affaire", "Qing (affectivité, relation) » existe toujours même si les affaires ne marchent pas. Et la logique ou les règlements pourraient être souvent négligés ou mal observés pour des raisons de «Qing». Tandis que pour les Occidentaux les affaires sont les affaires, l'amitié c'est l'amitié, ces deux traits de caractères sont aussi distincts que l'eau et le feu. Et les règlements ou les lois passent pour des tabous fermes comme la marbre. La remarque de ce PDG français témoigne, pour ainsi dire, d'une des différences typiques entre ces deux cultures.

5 En effet, dans une enquête faite par un chercheur américain sur l'interculturel, l'Occident et l'Orient se trouvent aux extrémités d'un schéma indiquant les différences culturelles, correspondant bien à la différence géographique: est-ouest - deux bouts du monde. Quelles sont alors les différences entre ces deux bouts ? Pourquoi ces différences? Est-ce qu'on peut les effacer sur-le-champ? Quelques évocations historiques, géographiques, linguistiques et même architecturales pourraient nous apporter des éclaircissements.

Citons toujours l'exemple de "Qing» du point de vue historique. Depuis longtemps "Qing " est hautement apprécié et se met toujours avant tout comme la première norme morale sociale et humaine. D'après Confucius, sans le Qing, on ne peut ni manger, ni 
dormir, ni parler, ni agir. Paradoxalement, «li » et «fa » sont souvent mal compris et négligés, quelquefois remplacés par la force des «héros»: vouloir toujours motiver la conscience de l'homme en prenant de bons exemples au lieu d'appliquer strictement une loi ou une logique à base des intérêts de tous. Donc il restait un espace pour une loi rigoureuse, laquelle a été facilement remplacée par la volonté de "quelqu'un ». La Chine a vécu longtemps sous le régime du féodalisme. L'empereur, c'est la loi. Une anecdote typique en fait la démonstration. Un jour, quelqu'un a amené un âne dans la cour, l'empereur l'a vu. Comme ce dernier ne connaît pas l'âne, il le prend pour un cheval. Les mandarins présents savent bien que leur maitre a commis une erreur, mais aucun n'ose dire la vérité. Et tout le monde suit l'empereur et dit que c'est un cheval. Car, la parole de l'empereur est obligatoirement juste malgré tout, ceux qui ne reconnaissent pas ce fait seront tués. Et la condamnation, dans ce cas, s'applique souvent jusqu'aux parents lointains. La loi représente la volonté d'un maître, dépend donc de quelqu'un, et peut changer avec ce quelqu'un; rien n'est bien défini. Ce "quelqu'un» est très important. D'un côté, l'autorité aveugle règne facilement, d'un autre, la parole de quelqu'un est souvent décisive. Dans ce cas-là, les relations avec des "quelqu'un" apparaissent comme primordiales. La volonté de l'homme se met en réalité avant la loi. Le «souverain » est tellement autoritaire que ses «ministres» sont obligés de s'exprimer par le détournement au lieu de prendre des risques de perdre leur tête. Cette tradition a malheureusement été transmise avec d'autres patrimoines de génération en génération jusqu'à nos jours. À l'heure actuelle, on reconnaît même pour les Chinois qu'il faudrait changer cette situation en établissant un ordre social fondé sur les lois et les règlements; beaucoup de chercheurs réclament ce changement, beaucoup de critiques vont contre cet ordre traditionnel: changer de l'ordre de « Qing-Li-Fa » en « Li-Fa-Qing » et valoriser la qualité tout en rejetant le défaut pour la réalisation de modernisations. Mais dans la pratique et dans la réalité, il est difficile de changer d'un seul coup et de déraciner ces habitudes acquises depuis plusieurs siècles.

D'après Confucius, les différences culturelles sont imputables souvent aux configurations géographiques : ceux qui vivent des montagnes, sont du type de «ren» (bienveillance, affectivité), ils préfèrent les montagnes, le calme, la nostalgie et vivent dans le passé en espérant la longévité; ceux qui vivent de la mer, sont du type de "zhi» (savoir, pragmatisme), ils préfèrent la mer, le déplacement, le loisir et vivent dans le présent, en jouissances. Ce que Confucius avait analysé il y a plus de deux mille ans, prévoyait bien justement les différences actuelles entre la Chine et la France. La Chine est plutôt un pays continental, donc les Chinois donnent plus d'importance à « ren », ils sont plus silencieux, introvertis et rétrospectifs; la France est plutôt un pays atlantique, donc les Français donnent plus d'importance à « zhi », ils aiment les déplacements et les changements, plus extravertis, ils profitent bien de l'instant présent. Ces deux types de culture ont chacune de son côté des qualités et des défauts. Celle de «ren » est plus humaine, plus réfléchie, plus profonde, tandis que celle de " zhi », plus pratique, plus moderne, plus créative. Mais avec le progrès de l'humanité, avec toutes sortes de moyens de transport, de communication, et avec les échanges multiples, la constitution géographique ne devient plus à cette époque un facteur important, et ces deux types de cultures ont donc tendance à voir évoluer leurs caractéristiques et se rapprocher, voire se compléter l'une l'autre.

Nous pouvons trouver aussi l'explication de ces différences à travers d'autres aspects linguistiques. Pour le mot «ren» que nous avons mentionné plus haut, Confucius a fait une analyse sémantique : «ren » est composé en caractère chinois de deux parties : deux 
+ personne = ren, « ren » est alors « deux personnes liées », le mot même contient déjà les relations étroites.

On observe que l'adresse inscrite sur l'enveloppe du courrier en Chine commence par le pays, suivent ensuite et dans l'ordre la ville, la rue et le nom du destinataire, tandis qu'en Occident, notamment en France, c'est tout à fait le contraire et l'on finit par le pays après avoir inscrit le nom du destinataire, de sa rue et de sa ville. Pour un livre, les Chinois préfèrent mettre la table de matière au début, en sommaire, alors que la plupart des occidentaux, les Français entre autres, la mettent à la fin. La présentation d'une enveloppe ou d'un livre est seulement un signe, un symbole, ou une convention, mais elle représente en profondeur les différences de modes de pensée : microscopique, analytique, moins contextuel et scientifique pour les Occidentaux et macroscopique, synthétique, plus contextuel, et littéraire pour les Chinois. D'où le fait que les Français abordent souvent, lors des réunions, le sujet directement, tandis que les Chinois mettent toujours un « chapeau » d'introduction pour présenter les contextes comme prologue; pendant la réunion, les Chinois préfèrent considérer les problèmes dans les grandes lignes alors que les Français entrent facilement dans les détails; les Chinois sont doués pour manier « l'ambiguïté », tandis que les Français ne comprennent que l'énoncé bien clair...

Il en est de même pour l'individualisme français et le collectivisme chinois. Le mot "propriété » se retrouve souvent dans des documents, tant elle est importante pour l'individu en France; tandis que pour les Chinois c'est l'intérêt du groupe qui compte le plus : le comportement de quelqu'un pouvait impliquer toute la famille jusqu'aux parents très lointains - un rebelle pourrait provoquer la mort d'un millier de parents - et inversement un honneur peut apporter aussi la gloire à la communauté. Donc, tous les comportements sont strictement observés par les autres, et l'idée de penser non seulement à soi-même mais surtout d'observer les rites et conventions d'une "famille » est ancrée dans la tête des gens. Il en résulte que les liens entre des individus sont extrêmement étroits. On a l'habitude de veiller, de bonne foi, aux autres: de communiquer avec les autres, d'aider les autres et de penser pour les autres.

Lors des visites en Chine, on peut constater qu'il y existe encore bon nombre de cours carrées comme dans les habitations traditionnelles, et les temples qui, toujours ont trois entrées horizontales successives.

12 Tandis qu'en France, les églises et les habitations traditionnelles sont construites verticalement à plusieurs niveaux montant vers le ciel. Ces différences architecturales, puisqu'elles regroupent tous les arts d'une culture, nous donnent également quelques suggestions. Dans une cour traditionnelle chinoise, souvent plusieurs familles vivent ensemble, on partage un même puits, une même cour, on y mange, on y discute... Beaucoup d'activités de plusieurs familles s'y déroulent ensemble. Dans ce cas-là, on vit quasiment en collectivité et les liens entre les gens sont très étroits. Ces conditions constituent une culture du temps " polychrone ", ne permettant pas la confidentialité. On raisonne les choses d'une façon plus perceptible et intuitive, de type « rond " comme disent certains chercheurs. Dans les immeubles à étages en Occident, c'est bien différent : on vit en famille, on est séparé par les murs protégeant ainsi les confidences, chaque salle a sa fonction, formant au fur et à mesure une culture du temps « monochrone». On traite les choses d'une manière déductive et raisonnable, du type dit « linéaire ».

13 Les évocations historiques, géographiques, linguistiques et architecturales montrent que les différences culturelles sino-françaises sont générales, profondes et n'ont pas été faites en un seul jour. Penser faire disparaître ces différences du jour au lendemain n'est 
sûrement pas une idée réaliste. Tout comme dit cet ancien PDG de Peugeot dont nous avons parlé au début de cette partie, on touche ici à des différences de mentalités, de modes de pensée et de systèmes de valeurs. Il est assez facile de dissiper les différences de signes, de symboles, puisque ce sont les couches superficielles de "l'oignon $»^{3}$ mais bien plus difficile de faire disparaitre celles qui sont au cœur. Ces différences sont telles que la coopération et le fonctionnement dans des entreprises sino-françaises demandent un grand effort. Comment faire face alors positivement à ces différences au lieu de s'en désespérer et de ne prendre aucune disposition particulière ? Comment peut-on " joindre ces deux bouts » du monde? Voilà une question difficile mais inévitable pour tous ceux qui s'impliquent dans les contacts sino-français.

\section{Approche : « he er bu tong »}

14 Il est naturel que la confrontation de deux cultures provoque des conflits et des problèmes. Et que l'imprégnation et la fusion entre des cultures soient très limitées. Certaines différences existeront pendant une période assez longue. Les échanges sont irréversibles, la coopération imposée mais les différences difficiles à faire disparaitre. Comment pourrions-nous faire pour traiter les différences qui persisteront?

Une approche " he er bu tong ", que nous avons puisée de la philosophie chinoise, paraît raisonnable et bien applicable, «he », c'est s'unir, s'harmoniser, « er » - mais, «butong » - différences. "He er butong», cela veut dire coexister, s'harmoniser avec des différences.

16 À l'origine du fameux livre du grand penseur, philosophe et éducateur chinois Confucius Lunyu (discussions) se trouve le dicton suivant: "Junzi he er butong, xiaoren tong er buhe ». (L'homme de bien vit avec des différences, l'homme de peu vit mal avec des ressemblances). Ici, Confucius loue les gens de qualité qui peuvent s'entendre bien malgré les différences, et méprise les gens de niveau bas qui ne peuvent pas s'entendre même s'ils sont du même genre. Pas seulement Confucius, Laozi a également traduit ces idées à travers sa doctrine $d u$ " yin » et du "yang ». D'après le taoïsme, yin et yang sont deux pôles totalement différents, mais constituent ainsi un couple inséparable : coexister dans un même univers l'un contre l'autre et se compléter l'un par l'autre. En plus, pendant la longue histoire chinoise, les esprits de différentes écoles, du confucianisme, du taoïsme, du bouddhisme et de la loi, en dépit des différends, coexistent en s'échangeant et en se complétant jusqu'à nos jours et nous laissent une épreuve par ces « junzi $»^{4}$ mêmes de ce " he er butong ».

En effet, cette philosophie dialectique existe également en Occident. Citons un exemple linguistique. En français, le mot «contre» contient aussi cette philosophie dialectique : dans le sens concret, "contre » signifie appuyé sur - il est contre le mur; mais dans le sens abstrait, "contre » - signifie s'opposer à - on lutte contre la drogue. De même, quand on parle de la mondialisation des cultures, de la coopération interculturelle, on n'efface jamais l'importance et la réalité des variétés, et l'on prend en compte l'échange, le rapprochement, le mélange et le cosmopolitisme des cultures. Le monde unique n'existe pas, il ne serait d'ailleurs pas viable. Dans la vie courante, on rencontre souvent ce genre de cas : un couple de caractères différents arrive à bien s'entendre; mais il est difficile pour un couple de tempéraments passifs de communiquer fréquemment, et il est rare que deux bavards s'écoutent. 
18 L'histoire nous apprend que les chocs entre deux cultures revêtent trois formes différentes: le premier cas, c'est une culture qui extermine et remplace une autre; le deuxième, c'est une culture qui domine une autre; le troisième, c'est une coexistence pacifique et amicale des deux cultures. Ce « he » désigne sûrement d'abord le troisième cas, mais pas un " he » inégal. Il n'existe pas une culture supérieure à une autre, chaque culture a ses qualités et ses défauts. $\mathrm{Ce}$ « he » doit se baser sur un respect mutuel. Pour la coopération sino-française, tout comme le dit Alain Pereyfitte dans La Chine s'est réveillée, la France a besoin de la Chine comme la Chine a besoin de la France. La Chine souhaite l'introduction de nouvelles technologies et la France recherche un grand marché et une main d'œuvre peu onéreuse. L'échange a supplanté la colonisation.

Bien entendu, malgré l'énorme diversité des esprits, on peut toujours trouver dans ces cultures une structure commune qui sert de point de départ à une compréhension et à une coexistence: la nature humaine qui se place au niveau de base pour «la programmation mentale humaine " ${ }^{5} \mathrm{Il}$ y a 27 ans lors d'un stage à l'exposition industrielle de France à Beijing, un ingénieur français nous a parlé de ce sujet en ces termes : « Il a dessiné un mont et deux hommes en train d'escalader des deux côtés. Il a dit: ces deux hommes signifient la Chine et la France, les deux pays veulent atteindre un même sommet, chacun d'un côté différent, d'une manière différente. Les méthodes et les itinéraires différent aussi, mais le sommet, le but est le même : la richesse et le bonheur de l'humanité. »Cette scène de discussion, si simple, symbolique et intéressante reste toujours dans notre mémoire. Le temps change, l'image change aussi : aujourd'hui, les deux hommes se réunissent d'un même côté et continuent à grimper au sommet. Quelle que soit la culture, quelles que soient les différences, le point de départ et le but sont identiques pour toute l'humanité : la recherche de la beauté, de la richesse, du bonheur et du perfectionnement. Tout ce qui est positif, actif, raisonnable et scientifique sera conservé et valorisé, tout ce qui est négatif, régressif, dépassé et retardataire sera rejeté et éliminé.

Il est normal que les gens se plaignent des difficultés dans ce « he ». Certains ont même échoué dans cette coopération. Un PDG français est finalement pris pour persona non grata par ses collaborateurs chinois et renvoyé par son supérieur français; des directeurs chinois sont mutés de poste de travail : ils n'admettent pas les différences et négligent l'existence d'une autre culture. Il est vrai que ce « he » a besoin d'efforts considérables et les vertus demandent toujours plus d'efforts que les vices. Ces efforts seront moins pénibles pour ceux qui ont plus de conscience, de motivation, de courage et de volonté. Un ingénieur français et son épouse déclarent avoir très bien vécu les quatre années de leur présence en Chine malgré l'obstacle de la langue : ils communiquaient fréquemment et amicalement avec des Chinois par des gestes, des expressions, des sourires. Chez eux, plus de compréhension que de malentendu, plus de réussite que d'échecs. Ils considèrent que leur travail et leur séjour dans une entreprise en Chine comme une occasion précieuse, une aventure intéressante et une expérience enrichissante.

21 Dans la pratique interculturelle, on sait déjà plus ou moins bien appliquer cette approche de « he er butong». À titre d'exemple, nous dressons ci-dessous un schéma structural typique de l'organigramme (voir schéma $\mathrm{V}$ ) dans beaucoup d'entreprises sino-françaises installées en Chine. Il exprime en quelque sorte cette approche de la coexistence et de la complémentarité. A travers ce schéma on pourra découvrir une première approche de l'harmonie « he». 


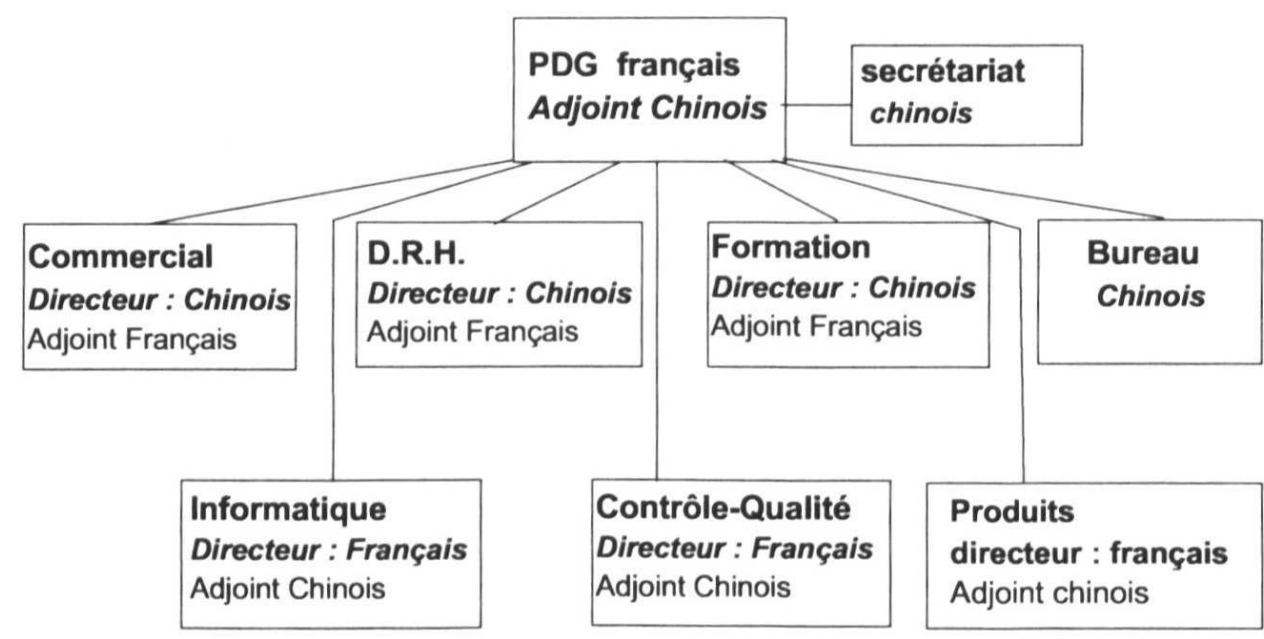

Le schéma $\mathrm{V}$ expose une particularité évidente : la partie chinoise connaissant plus le milieu s'occupe plus du côté personnel et relationnel, tandis que la partie française plus forte en technologie et en gestion assume la qualité et ce qui concerne la production. Ainsi chaque côté valorise de son mieux sa qualité. Si « he er butong » pouvait bien être compris et pratiqué, si l'on avait bien conscience de profiter des qualités des deux cultures et valoriser convenablement leur efficacité, les entreprises interculturelles pourraient mieux réussir malgré les problèmes puisqu'elles regroupent deux « génies " de l'humanité au lieu d'un seul.

Toujours est-il que ce « he er butong » reste une approche dont le but est de réaliser les projets communs en conquérant de plus en plus de " tong " et une meilleure coexistence. $\mathrm{Au}$ début, les deux partenaires ne se connaissent et ne se comprennent pas bien. Mais dans ce "he " avec des différences, on se connaît de mieux en mieux et on pourra atteindre une meilleure compréhension et une meilleure coordination. Dans ce cas-là, accepter l'approche de "he er butong ", reconnaître les différences, les comprendre et savoir vivre comme «junzi » ces différences, c'est le seul chemin qui nous mène à la réussite.

\section{Enjeux : contextes, qualités, managers}

Il est clair que la coexistence de deux cultures est bien différente de celle de deux individus. Quelques enjeux nous paraissent non négligeables et méritent notre considération.

Marc Lipiansky a bien insisté sur le rôle important des " contextes » dans son brillant exposé sur la communication interculturelle ${ }^{6}$ : selon le contexte dans lequel elle se situe, la communication peut avoir des caractères très différents. Sans doute, le cas de l'entreprise interculturelle se différencie-t-il de celui d'une personne immigrée puisque la première concerne plutôt la coopération de deux entités culturelles juxtaposées tandis que l'autre doit toujours s'intégrer dans la culture du pays d'accueil ${ }^{7}$ Pourtant, l'environnement $\mathrm{du}$ pays où s'installe l'entreprise conditionne grandement son fonctionnement malgré la juxtaposition. Sur ce point, une entreprise pourrait être prise, pour ainsi dire, pour un immigré agrandi et renforcé. Justement, le PDG mentionné ci- 
dessus a intelligemment commencé par se taire et écouter ${ }^{8}$ pour « mieux comprendre » et " mieux réagir ». Car « la compréhension, le respect et la tolérance sont le secret de l'art d'aplanir les différences culturelles » et « les bons réseaux de relations en Chine (appelés : guanxi) sont le facteur de la réussite $"$.

Un autre PDG, d'une entreprise sino-française installée dans une petite ville de Chine, nous a expliqué un problème qu'il rencontre souvent dans son travail : la disparité des réactions des collègues chinois - certains acceptent facilement de nouveaux concepts occidentaux tandis que d'autres restent obstinés dans les concepts du $18^{\text {ème }}$ ou $19^{\text {ème }}$ siècle. Cela le gêne beaucoup : il lui est difficile de comprendre et de distinguer et traiter des cas différents. Pour bien décrire ce problème, ce PDG a même fait un schéma et donné ses réflexions.

La disparité dans tous les pays en développement est générale et inévitable. Car ces pays vivent une période de transition: de tradition en modernisation dans tous les sens et à tous niveaux. Plus le rythme est rapide, plus la disparité est impressionnante. En Chine, par exemple, on peut voir le pousse-pousse à pédales et la BMW circuler sur un même boulevard et de vieux " hutong " $^{10}$ à côté de luxueuses Galeries Lafayettes... de même, les idées de l'époque de Qing (1644-1911), de Sun (début de ce siècle), de Mao (1921-1976) et de Deng (depuis 1978) coexistent, se mélangent durant cette époque de transition. Ce phénomène tout à fait normal pour un Chinois est en général difficilement compréhensible par les collaborateurs français.

Chaque pays a ses contextes particuliers. Comprendre et s'adapter à l'environnement constitue un enjeu majeur pour la réussite de la coopération. Pour un pays de "Qing ", les relations restent encore pour le moment la priorité ; pour une entreprise ayant peu de moyens, il est difficile de moderniser tous les équipements en peu de temps; pour un système socialiste, la présence du Parti au pouvoir (même si cette présence est secondaire dans ces entreprises) doit être plus ou moins respectée... Ces compréhensions et appropriations ne font que servir à une facilité de la coexistence et de la coopération. Comme le disent beaucoup de chercheurs européens: "En retour, une meilleure compréhension de l'éthique asiatique chez les occidentaux devrait rendre les relations plus fécondes et conduire, sans inféodation, à l'invention d'un nouveau modèle de modernité ».11

Alors que leur partenaire français fait attention à écouter et à comprendre, la partie chinoise dans l'ensemble est prête aussi à apprendre la gestion scientifique et les nouvelles technologies et techniques de l'Occident : les "qualités » jouent et deviennent un autre enjeu. La réalisation des quatre modernisations ${ }^{12}$ en Chine a besoin de l'introduction de l'Occident. Pendant une longue période dans l'histoire, les Chinois furent blessés par l'agression de l'Occident et des étrangers, puis certains se tournèrent vers l'admiration aveugle pour l'Occident, maintenant on décide sagement d'apprendre auprès de l'Occident en dépit des douleurs historiques. Car Confucius et Mao ont tous encouragé les Chinois à "tirer la bonne partie des autres pour s'enrichir ", et Deng a annoncé aussi cet appel connu " peu importe que le chat soit blanc ou noir, il est toujours bon s'il attrape bien les rats ». Donc en principe, dans les entreprises sino-françaises en Chine, l'application de la gestion scientifique et l'introduction de nouvelles technologies ne se mettent point en question, l'occidentalisation va jusqu'au costume. L'occidentalisation ne devient plus un défaut, mais une mode pour tout le monde au moins dans la parole; l'occidentalisation signifie souvent la modernisation. L'humanité sait bien choisir ce qui est bon en le valorisant et rejeter les déchets. Il ne manque pas de 
bons exemples de ce genre dans l'histoire de l'humanité: la Grèce s'est inspirée de l'Egypte et Rome a tiré profit de la Grèce.

Bien sûr, la seule occidentalisation n'est pas suffisante; inéluctablement pour chaque pays, il existe un problème de nationalisation et d'appropriation : un produit introduit doit toujours s'adapter au climat du pays pour prospérer. Nombre de McDonalds et de Carrefour installés en Chine ne manquent pas d'adopter certaines caractéristiques nationales chinoises : beaucoup plus d'employés et de clients, un goût nuancé et des prix différents...

31 Comme l'introduction de l'occident devient une mode en Chine, on profite de toute occasion pour apprendre auprès de l'Occident. Chez Dongfeng-Citroën où $70 \%$ de capitaux sont investis par la Chine, le PDG est un Français. Dans cette entreprise, tous les travailleurs doivent s'habiller correctement, comme en France, même le service au restaurant de l'entreprise est francisé ou occidentalisé. La présence d'un "manager " compétent est en quelque sorte un facteur décisif pour la réussite d'une entreprise interculturelle. Ces managers doivent avoir non seulement l'expérience de la direction d'entreprise dans leur pays, mais surtout la conscience de l'adaptation nécessaire à la gestion d'une entreprise interculturelle. Nous observons que tout directeur d'entreprise sino-française est peu ou prou un spécialiste de pratiques interculturelles, car son travail l'oblige à réfléchir et à traiter tous les cas. Bon nombre de PDG ont même concrétisé leurs recherches par la publication de travaux fondés sur leurs propres expériences.

\section{Modèle de « mohe » : trilogie}

La coexistence des deux cultures dans une coopération internationale finira inévitablement, tout comme dans un couple, par parcours commun de plusieurs étapes de "mohe ». Mais ce "mohe » sera plus difficile et plus compliqué. Il est important, dans le modèle de «mohe » que l'on cherche, de remarquer cette «trilogie »: « he » - « mo »" he ». C'est en effet un cycle de trois phases à vivre : commencer par " he", passer par «mo » pour atteindre un autre « he ». Pourquoi commencer par « he » et qu'est-ce que veut dire ce «he»? Est-ce que ces deux «he» sont les mêmes? Comment faut-il comprendre ce modèle?

Regardons d'abord le premier " he ». Il est, pour ainsi dire, dans la plupart des cas formel : « he » de capitaux, d'organisations, de personnel... Après plusieurs négociations, les deux partenaires trouvent les intérêts communs donc signent leur accord. Alors deux entités se recomposent en une et réalisent leur premier «he ». L'entreprise est toute nouvelle, les employés en tenue correcte, l'aménagement bien réalisé et le fonctionnement normal. Mais pourtant la partie française ne comprend pas pourquoi ses collègues chinois consacrent autant de temps en réunions longues et nombreuses, et les Chinois ne sont pas satisfaits non plus des candidats proposés par leurs collègues français... Ce «he » n'est nulle part solide, prévoit énormément de différends et de conflits. Cette entité apparemment unique est clairement divisée en deux parties. Pourtant cette étape initiale est importante.

Une fois ce «he» fini, le «mo» suit. Cette étape de conflits durera beaucoup plus longtemps en passant tout un processus de « moudre » : de formes aiguës en celles rondes jusqu'aux formes lisses, bien mélangées. 
Ce «mo» n'est toutefois pas un statu quo, il entre tout de suite en mouvements et progressions: négociation, appropriation, conflits. Dans ces confrontations, il est important que les deux parties tiennent compte de l'existence du partenaire pour trouver une réconciliation harmonieuse. Nous avons formulé ci-dessous, en nous référant aux théories psychologiques de l'harmonie, de l'équilibre et de la coordination, un tableau de 36 carreaux afin de repérer les points correspondant aux possibilités de conformité entre les deux partenaires.

Tableau de 36 carrés ou repères de conformité

\begin{tabular}{|c|c|c|c|c|c|c|}
\hline B. & volontaire & conscient & possible & inccuscient & involontaire & impossible \\
\hline volontaire & vert & vert & vert & jaune-vert & jaune & rouge \\
\hline conscient & vert & vert & vert & jaune-vert & jaune & rouge \\
\hline possible & vert & vert & vert & jaune-vert & jaune & rouge \\
\hline inconscient & jaune-vert & jaune-vert & jaune-vert & rouge & rouge & rouge \\
\hline involontaire & jaune & jaune & jaune & rouge & rouge & rouge \\
\hline impossible & rouge & rouge & rouge & rouge & rouge & rouge \\
\hline
\end{tabular}

faciles, des zones interdites et des zones entre les deux. Quand l'on rencontre des problèmes, il faut bien savoir ce qui serait possible et faisable d'après les indices différents des deux côtés. Dans ce tableau, la zone verte et la zone rouge sont bien claires, faciles à passer : on réalise facilement ou on abandonne. Les problèmes se manifestent surtout dans des zones «jaune » ou «jaune-vert» où il existe plusieurs possibilités: si l'on peut bien les traiter, ou avec des méthodes adéquates, ou avec de la patience, les problèmes pourront être réglés, la coexistence paisiblement réalisée, et «mo » passé en « he ». Sinon, ce sera les échecs.

Après une période de coexistence, de "mohe ", on trouvera des aspects nouveaux dans ces entreprises : la compréhension est plus facile - on observe bien l'emploi du temps et on prend l'habitude de «rendez-vous » et on s'occupe des problèmes personnels aussi 
bien que de ceux du travail ; la convivialité est établie - l'humour n'est plus considéré comme la méchanceté et la critique nécessaire est acceptée ; on assiste aussi à la mise en place de projets plus efficaces et à un plus grand respect des règlements. Chaque partie a fait ses efforts. Ce deuxième " he » après l'étape « $m o$ ", sous forme de fusion au niveau profond, se montre bien plus solide.

Les échanges à ce moment-là sont plus profonds et efficaces. Du côté chinois, on applique consciemment des règlements, les ouvriers comprennent que la qualité du travail est la vie de l'entreprise, ils apprennent assidûment les nouvelles techniques, les directeurs raccourcissent leurs réunions ; et les ingénieurs français de leur côté ne se contentent pas seulement de l'efficacité du travail, mais également font des efforts en donnant des explications nécessaires. Le respect et la confiance sont ainsi établis et le dialogue s'établit. Dans ce cas-là, il ne manque ni de gestion scientifique et rigoureuse, ni de bonne qualité, ni d'ambiance de solidarité. Dans le travail, on est sérieux et efficace; en weekend, on organise les soirées de danse et de karaoké. Il y a sans doute eu conflits et problèmes dans la coopération, mais aussi vitalité et réussite. Une nouvelle culture d'entreprise est née, basée sur les qualités des deux, $1+1=3$ devient la réalité. Pourtant la trilogie «he» - «mo» - «he » ne s'achève pas, elle se renouvelle d'une façon plus approfondie.

\section{Conclusion}

Depuis plus de vingt ans, des milliers d'entreprises françaises ont établi des relations avec la Chine. Certaines ont réussi, d'autres ont échoué. À part des raisons économiques et/ou politiques, la communication ou le management interculturel jouent un rôle décisif. Dans cette coexistence multiculturelle, notamment de deux cultures si différentes, il se vit une période difficile de «mohe » tout comme pour un ménage. Pour faire face positivement à ces différences, une approche est proposée: «he er bu tong» tirée de la pensée traditionnelle chinoise. Cette coexistence est pourtant bien différente de celle d'un ménage. La nouvelle problématique nous montre trois enjeux importants: contextes, qualités et managers.

Les faits prouvent que la coexistence des entreprises sino-françaises est bien possible et que le "mohe » n'y est pas un mirage à condition de faire des efforts. Le processus de «mohe " pourrait être « une trilogie recyclable»: « he " « mo » - « he ». Le premier " he » est plutôt formel, après une étape de "mo », le «he » devient plus profond et solide. L'important pour les deux partenaires dans cette trilogie de "mohe», c'est de tenir compte de l'existence à tout moment d'une autre culture et d'agir conformément aux intérêts des deux selon le « tableau de 36 carrés » proposé. Les succès couronneront d'autant plus ceux qui ont plus de détermination, de conscience et de pratique. 


\section{BIBLIOGRAPHIE}

BENOIT, D. et coll., Introduction aux sciences de l'information et de la communication. Paris : Editions d'organisation, 1995, 414 p. BOUCHER, F., Travailler avec les Chinois. Paris : Nathan, 1995, 126 p. CAMILLERI-MARGALIT, C, COHEN-EMERIQUE (Sous la direction de), Chocs de cultures, Paris : L'Harmattan, 1989, 398 p.

CHASLE, R. (Sous la direction de), L'alternance culturelle, Editions Publisud, 1994,316 p.

CHEVRIER, S., Le management des équipes interculturelles, Paris : Puf, 2000,211 p.

DTRIBARNE, P. et coll., Cultures et mondialisation, Paris : Editions du Seuil, 1998,355 p.

HALL, E.T., Le langage silencieux, traduit de l'américain, Paris : Editions du Seuil, 1984, 237 p.

INGLEHART, R., La transition culturelle, Economica, 1990, 576 p. JIA, Y., La théorie de la communication interculturelle, Shanghai (Chine) : Editions des Etudes et des Recherches des langues étrangères, 1995, 525 p. KALTENMARK, M., La philosophie chinoise, Paris : Puf, 1972, 122 p. LARRY, A., Samovar et coll., Understanding intercultural communication, Wadsworth Publising Co, 1981, $286 \mathrm{p}$.

LECLERC, G., La mondialisation culturelle, Paris : Puf, 2000, 486 p.

LE DAIYUN, LE PICHON, A., Dialogue transculturel, Shanghai (Chine) :

Editions culturelles de Shanghai, 1998, 199 p.

LEMAIRE, P-M., Communication et culture, Québec : Les presses de l'Université Laval, 1989, 296 p.

LING, D., Etudes sur la communication interculturelle, Fujian (Chine) : Editions de Xiamen, 1994, $297 \mathrm{p}$.

PEILL-SCHOELLER, P., Interkulturelles Management, Springer-Verlag Berlin Heidelberg, 1994, 288 p. (traduction en chinois) PEYREFITTE, A., La Chine s'est réveillée, Paris : Fayard, 1997, 431 p. SFEZ, L., Dictionnaire critique de la communication, T. 1, Paris : ADAGP, 1993,922 p.

TROMPENAARS, F., L'entreprise multiculturelle, traduit de l'américain, Maxima, 1994, 308 p.

WARNIER, J-P., La mondialisation de la culture, Paris : La découverte, 1999, 120 p.

\section{NOTES}

1. "Joindre les deux bouts » est une expression française qui se rapporte à l'économie, nous l'empruntons ici plutôt de son sens propre : lier les deux bouts - l'est et l'ouest du monde.

2. Cf. Li-Fa-Qing et Qing-Li-Fa, «Paris Match » Numéro spécial En Chine, p. 51.

3. cf. Geert Hofstede Vivre dans un monde multiculturel, p. 24. D'après lui, il existe quatre niveaux différents de manifestation d'une culture: symboles, héros, rituels et valeurs. Ces différents niveaux sont figurés par les « pelures d'oignon ».

4. Junzi : intellectuel et gentilhomme, ou homme de bien

5. cf. Geert Hofstede, Vivre dans un monde multiculturel, p. 21. L'auteur divise la programmation mentale humaine en trois niveaux dont le premier, la base, est la nature humaine.

6. Cf. Denis Benoît et coll., Introduction aux sciences de l'information et de la communication, p. 214. 
7. cf. Hugues Hotier, Les cultures nationales face aux entreprises multinationales. Entreprise et communication, actes du $2^{\mathrm{e}}$ séminaire interculturel sino-français de Canton », p. 16.

8. cf. Madelaine Bardier-Decrozes, Le reportage sur Montgolfier, Paris Match, numéro spécial En Chine, p. 50.

9. cf. Elaboration d'une stratégie fructueuse en vue d'une négociation efficace de contacts avec les Chinois, Internet, site: Perspectives sur la Chine, expansion du commerce (Ministère des Affaires canadiennes).

10. Hutong : petites ruelles traditionnelles à Beijing

11. Cf. Le temps de l'Asie (Megatrends in Asia, John Naisbitt, Nicolas Brealey éd. Londres, nov.1995. Résumé en français par Rémi Perelman (janvier 1996).

12. Quatre modernisations : modernisations industrielle, agricole, scientifique et technique, de la défense nationale.

\section{RÉSUMÉS}

Dans la coexistence multiculturelle en entreprise, il se vit une période initiale d'adaptation parfois difficile, de "mohe", tout comme pour un jeune couple. Pour faire face positivement à ces différences, une approche est proposée : « he er bu tong ", tirée de la pensée traditionnelle chinoise. Cette nouvelle problématique met en relief l'importance de trois facteurs: contexte, qualité et managers. Les faits prouvent que le «mohe » n'est pas une chimère à condition que les parties en présence fassent les efforts nécessaires. Le processus de « mohe » aboutissant alors sur une trilogie d'étapes d'adaptation, recyclables. Le monde de yin et de yang est plein de contradictions, mais c'est justement dans les «mohe » que nos entreprises interculturelles, voir notre société, pourront progresser.

In a multicultural co-living context in a company, there is a period of adaptation that could be sometimes difficult, called "mohe ", like for a new couple. To face positively these differences, an approach taken from the traditional Chinese thinking, is proposed: " he er bu tong ». This new issue enhances the importance of three stakes : context, quality and managers. Facts prove that "mohe" is not an illusion, if present, counterparts do required efforts. The "mohe "process results in three stages of recyclable adaptation. The "yin and yang " world is full of contradictions, however it is truly in the "mohe» that intercultural companies, maybe our society too, may evolve progress.

\section{INDEX}

Mots-clés : entreprise, interculturel, mohe, sino-française

\section{AUTEUR}

\section{ZHIJIE WANG}

WANG Zhijie, Docteur en sciences de l'information et de la communication (France) et professeur titulaire à l'Université de Wuhan (Chine). Depuis 1997, des recherches axées 
sur les thèmes de la communication interculturelle, notamment de celle dans les entreprises à capitaux mixtes en Chine. Travaux récents (depuis 2000) : contribution à l'étude comparative oriento-occidentale dans la presse et l'information d'entreprise ; $1+1$ $=3$, est-ce possible $?$ nouvelles réflexions sur la communication interculturelle dans les entreprises sino-françaises; si la mondialisation d'économies est irréversible, celle de cultures le sera aussi ; un parcours rapide sur les recherches de la communication en France. 